\title{
Exploration didactique de la langue du milieu professionnel à l'université : quel apport pour la définition de tâches d'enseignement- apprentissage?
}

Marie-Christine Deyrich

\section{CpenEdition}

\section{Journals}

Édition électronique

URL : http://journals.openedition.org/asp/1106

DOI : 10.4000/asp. 1106

ISBN : 978-2-8218-0396-1

ISSN : 2108-6354

\section{Éditeur}

Groupe d'étude et de recherche en anglais de spécialité

\section{Édition imprimée}

Date de publication : 1 mars 2004

Pagination : 125-134

ISSN : 1246-8185

Référence électronique

Marie-Christine Deyrich, «Exploration didactique de la langue du milieu professionnel à l'université : quel apport pour la définition de tâches d'enseignement-apprentissage ? », ASp [En ligne], 43-44 | 2004, mis en ligne le 01 décembre 2004, consulté le 19 avril 2019. URL : http://journals.openedition.org/ asp/1106 ; DOI : 10.4000/asp.1106

Ce document a été généré automatiquement le 19 avril 2019

Tous droits réservés 


\title{
Exploration didactique de la langue du milieu professionnel à l'université : quel apport pour la définition de tâches d'enseignement-apprentissage?
}

\author{
Marie-Christine Deyrich
}

\section{Le monde professionnel et ses délimitations pour l'enseignement de la langue de spécialité}

\subsection{Rencontre du « milieu professionnel » et de l'espace didactique}

1 La spécificité du langage des différents « milieux professionnels » a généré d'importants travaux qui nourrissent la réflexion didactique du secteur LANSAD. Dans la description des différentes langues de spécialité, la notion de milieu professionnel est particulièrement développée dans l'analyse des genres (Swales 1990), notamment parce qu'elle débouche sur une délimitation scientifique des communautés discursives et sur une identification de différents domaines de spécialité et des concepts qui leur sont propres (Resche 2003).

2 Dans le champ de l'enseignement-apprentissage, la langue de spécialité est facteur de motivation et de réussite : de nombreux travaux témoignent de l'utilité d'une référence crédible à un domaine de spécialité clairement défini (Mémet 2003). Dans ce type d'approche, souvent qualifiée d'intégrée, la notion de «milieu » professionnel n'a pas cependant pas été importée en l'état: elle a subi des transformations pour prendre en compte la spécificité de la situation. On ne peut en effet établir un lien direct entre, d'une part, le découpage d'une réalité professionnelle qui se voudrait objective, alors qu'elle 
dépend du choix du chercheur et, d'autre part, la définition d'objectifs correspondant aux besoins des apprenants (Widdowson 2003: 70-71). En effet, il n'y a en aucun cas adéquation entre les besoins langagiers du monde professionnel et ceux d'étudiants qui apprennent une langue étrangère en milieu institutionnel. Il s'agit là d'une situation totalement artificielle - même lorsqu'elle se fonde sur des documents et/ou des situations jugé(e)s « authentiques ».

3 À l'inverse, on observe des cas où l'existence même de la langue de spécialité est mise en cause. Dans les cursus universitaires des spécialistes d'autres disciplines, l'anglais est omniprésent mais cela n'implique pas pour autant que l'enseignement correspondant puisse établir des liens avec le domaine de spécialité. Dans certaines universités, l'expression «non-spécialistes » réfère encore au secteur LANSAD et parfois même, pour des raisons d'organisation ou de budget, on regroupe des "non-spécialistes" d'art plastique, d'AES et de psychologie, par exemple. Le besoin se définit alors en heuresannée plutôt qu'en contenus d'enseignement-apprentissage. À ce sujet, E. Aranda (2002 : 90) évoque le fait que les enseignants intervenant dans ces modules ne peuvent s'engager dans une analyse et une élaboration didactique, puisqu'ils sont déjà mobilisés ailleurs et ne sont donc pas en mesure de tenir compte de la spécificité du public. Or, la prise en charge didactique de la spécificité suppose une réflexion concertée.

Enfin, la langue de spécialité recouvre des situations où les liens avec le milieu professionnel s'avèrent parfois difficiles à établir. Nous nous référons ici à l'enseignement de la langue de spécialité en Administration économique et sociale (AES), formation pluridisciplinaire qui concerne un public relativement nombreux et qui débouche sur des profils de postes très diversifiés. Le champ d'investigation des milieux professionnels susceptibles d'être impliqués y est très largement ouvert et demande une réinterprétation des concepts dans leur mise en perspective.

\subsection{Du milieu au « paysage professionnel»}

Revenons sur la notion de «milieu » qui, au sens ordinaire du terme, définit ce qui est "entre» (au milieu de). Nous estimons que cette façon d'envisager le milieu professionnel lui confère une apparence statique qui ne tient pas compte des évolutions de la langue de spécialité et de son apprentissage. À l'inverse, parmi les nombreux domaines scientifiques où cette notion intervient, celui du géographe semble plus proche de la dynamique de notre domaine : le milieu y est défini comme un ensemble d'éléments d'ordre naturel et d'éléments sociaux, des infrastructures produites par les hommes, des institutions, des cultures, etc., le tout formant un système. Appréhendé ainsi, le milieu professionnel est donc à la fois naturel et culturel, à la fois subjectif et objectif, à la fois collectif et individuel.

D'autre part, les problématisations qui découlent de la notion de milieu demandent à être mises en perspective avec le travail des chercheurs du domaine LANSAD : ceux-ci portent un regard particulier sur le milieu qu'ils étudient, regard qui le délimite et lui donne sens. On glisse ainsi de la notion de milieu à celle de paysage, portion d'espace examinée par un observateur qui délimite ainsi un fragment de la réalité, fragment de la langue de spécialité pour ce qui nous concerne. Or, le paysage n'existe pas en soi, puisque c'est une invention historique, culturelle ou linguistique. Nous en déduisons qu'il paraît logique de concevoir le «paysage professionnel» comme une construction humaine, du double 
point de vue de sa face visible et concrète et de sa représentation par les acteurs professionnels.

7 La complexité du " paysage professionnel » résiderait alors dans une irréductible dualité, une tension entre l'objectivité du support matériel, d'une part, et la subjectivité du récepteur, d'autre part. De notre point de vue, le concept de paysage nous permettrait d'envisager à la fois le milieu (objet) qui se donne à voir : le discours professionnel et le construit de l'esprit, mettant donc l'accent sur l'interaction entre l'objet et les sujets.

En conséquence, deux aspects de l'objet langue professionnelle seraient ainsi mis en exergue : la variabilité, l'instabilité ainsi que sur son évolution au fil du temps et le rôle des représentations et des interprétations des différents acteurs.

Dès lors, il y aurait non seulement un lexique de spécialité, des discours spécifiques, des situations clairement identifiables du « milieu professionnel » mais aussi, en complément, les traces de l'interaction avec des énonciateurs dans des situation bien définies, dans un contexte professionnel ou institutionnel.

\section{Le paysage professionnel à l'épreuve du contexte didactique}

10 Nous reprenons ici la notion de paysage professionnel, telle que nous venons de la définir, pour la mettre en perspective avec le concept de transposition didactique qui met l'accent sur la gestion des écarts entre, d'une part, les savoirs et les pratiques du monde professionnel dans leur description scientifique et, d'autre part, les besoins spécifiques des apprenants (Deyrich 2001). Cette approche transpositive implique un profond travail de didactisation qui doit prendre en compte la spécificité du public d'apprenants avancés. Dans ce contexte, nous fondons les stratégies d'enseignement sur la notion de tâches d'enseignement-apprentissage (Ellis 2003) qui peut, selon nous, contribuer à une définition de stratégies adaptées et motivantes.

\subsection{Une interprétation spécifique de l'objectif communicationnel}

11 Nous ne reviendrons pas ici sur l'impossibilité matérielle de transformer nos étudiants en locuteurs parlant couramment l'anglais. Ce constat ne peut cependant conduire à un abandon pur et simple. Il est de ce fait important de donner à la communication une acception propre à la situation.

En effet, l'apprentissage de la communication est très exigeant puisqu'il repose notamment sur un mélange d'entrainement de type behavioriste avec la vélocité de plus en plus grande de la réaction devant l'imprévu et sur une mobilisation des connaissances, associée à un raisonnement rapide avec anticipation, adaptation, ajustement et précorrection d'erreurs. Or, dans les nombreux cas où les groupes sont de grande taille, les conditions matérielles sont telles qu'on ne peut organiser des jeux de rôle dans des situations réelles de communication, et plus particulièrement dans la langue de spécialité à l'université. Qu'en est-il alors de la communication? Que reste-t-il à ce moment-là qui puisse être une préparation à un stage que les étudiants effectueraient dans le pays étranger?

Bien évidemment, le problème ne peut être résolu s'il demeure posé en ces termes. Pour notre part, nous estimons qu'il est préférable de considérer que les étudiants se serviront 
de l'accumulation de méthode étudiée en cours pour traiter les problèmes ultérieurement, de façon différée. De ce point de vue, on peut considérer que leur « bagage » méthodologique devrait les aider à traiter les données correctement lorsqu'ils se trouveront dans une situation de communication au cours de laquelle ils devront se montrer efficaces.

\subsection{Propositions pour une définition de contenus}

Nous formulons l'hypothèse que l'approche didactique de la langue de spécialité doit viser un certain type de professionnalité en étant utilitariste à deux niveaux. Tout d'abord, nous convenons qu'il est nécessaire de situer les apprentissages dans le contexte professionnel en intégrant la théorie du genre comme le souligne D. Dressen Hammouda (2003: 81), mais nous ajoutons qu'il est alors indispensable d'articuler la définition de contenus avec les savoir-faire opérationnels dans des situations d'utilisation qui partagent certains aspects de la culture professionnelle. Le second niveau concerne l'utilité dans l'apprentissage : c'est elle qui, selon nous, doit primer et qui doit constituer le point de départ du cheminement transpositif. Nous en traçons ici les grandes lignes :

1. Identification des besoins des étudiants : il s'agit de déterminer quels savoirs devront être travaillés prioritairement pour améliorer leurs capacités de réception (compréhension écrite et orale) et de production (expression orale et écrite).

2. Examen de l'implication des quatre savoir-faire langagiers dans la perspective de la spécialité : le but est de donner une orientation professionnelle crédible et souple en fonction des besoins de communication repérés. Il s'agit donc d'une contextualisation prévisionnelle des savoir-faire qui se fonde sur les attentes de la communauté professionnelle cible.

3. Investissement dans la préparation pédagogique de ces savoir-faire (plus ou moins professionnalisés) et des savoirs qui y sont associés. La préparation de tâches d'enseignement-apprentissage tient compte de l'analyse menée en amont.

Dans cette démarche, il est indispensable que la projection dans l'avenir intervienne mais elle n'est que secondaire. Elle se donne pour objectif de fixer une orientation professionnelle générale, non pas un programme de formation. On retrouve ici une déclinaison de la notion de "paysage professionnel»: le découpage est forcément subjectif car il privilégie le regard didactique, délimitant ainsi un espace professionnalisé, dans lequel sont mis au premier plan certains besoins d'enseignement-apprentissage plutôt que d'autres. Cette sélection guide ensuite l'organisation des objectifs et les contenus.

\subsection{Illustration de la démarche : le cas de l'anglais de spécialité en AES}

\subsubsection{Identification des besoins des étudiants}

En nous appuyant sur les observations effectuées en cours, nous avançons que les besoins les plus urgents sont liés à l'insuffisance de pratiques langagières. En effet, les difficultés ne concernent pas prioritairement la syntaxe ou le lexique mais le contact direct avec la langue (capacités de réception) et la production d'énoncés suffisamment longs issus d'une véritable prise en charge énonciative (notamment en production orale). Nous en avons 
déduit que le travail doit donc porter davantage sur la fluidité, sur la prise de confiance et qu'il doit donner progressivement des moyens d'autonomisation.

\subsubsection{Examen de l'implication des quatre savoir-faire fondamentaux dans la perspective de la spécialité}

Pour interpréter l'orientation professionnelle de l'anglais dans la formation universitaire en AES, nous avons, en collaboration avec les collègues spécialistes des autres disciplines, tenté d'établir les grandes lignes de l'utilisation professionnelle potentielle des pratiques langagières. En Annexe 1, nous reproduisons un tableau qui résume notre interprétation didactique de ce paysage professionnel. Il tente notamment de décrire les caractéristiques de la visée communicative prospective de l'anglais de spécialité en AES: quatre orientations principales ont été dégagées en termes de pratiques langagières et pour chacune d'elles, on a relevé les savoir-faire qui sont mobilisés prioritairement.

Revenons brièvement sur la façon dont les savoir-faire sont impliqués dans ces pratiques professionnelles. Tout d'abord, les quatre savoir-faire sont représentés - un témoignage, si besoin était, de leur interaction dans la communication. On observe que la compréhension écrite y occupe cependant la première place dans la majorité des cas: c'est le savoir-faire langagier de base, celui dont tout étudiant doit maitriser les rudiments s'il veut utiliser la langue en contexte professionnel. D'autre part, on remarque une disparité entre l'oral et l'écrit (qui domine). Il convient toutefois de signaler que l'oral ne doit absolument pas être négligé. En effet, si l'on se réfère au seul cas où ce savoir-faire intervient au premier plan (participation à des événements professionnels), l'aisance présupposée ne peut être spontanée. Le niveau d'exigence est donc relativement élevé. On peut enfin en déduire que, pour que le niveau de maitrise soit satisfaisant pour la communauté professionnelle cible, il faudra développer des aptitudes en expression, car être compris ne peut suffire: la langue doit être d'une qualité réelle sur les plans lexical et syntaxique pour être pris au sérieux dans le monde professionnel. De la même façon, en compréhension, de réelles dispositions sont attendues, notamment pour une adaptation des stratégies à la diversité des situations et des supports ainsi que pour une réactivité se traduisant par une prise de position informée et un recul critique pertinent.

\subsubsection{Investissement dans la préparation pédagogique des savoir-faire sélectionnés et des savoirs qui y sont associés}

L'entrée sur la scène didactique suppose qu'il y ait eu transformation préalable des données, de telle sorte que l'activité des apprenants soit sollicitée activement en fonction d'une orientation dont ils perçoivent la cohérence. Ce dernier point est particulièrement important dès lors que l'on admet que la cognition est systématiquement située (Lave \& Wenger 1991), ou en d'autres termes que l'environnement influe sur le fonctionnement mental avec lequel il interagit. Dès lors, la didactisation doit inclure le contexte et, par conséquent, la communauté de pratique, qui jouent un rôle de médiateur dans l'apprentissage.

Il reste à envisager l'impact sur les stratégies d'enseignement d'une telle intégration de la cognition située. Cette approche exclut le recours à une succession d'exercices qui focaliseraient sur la forme au profit de tâches mettant l'accent sur la construction du sens (Ellis 2003: 3). Celles-ci englobent ainsi un ensemble d'objectifs visant à donner aux étudiants l'occasion d'utiliser la langue dans des mises en situation crédibles. De plus, les 
apprentissages visés sont fixés pour le groupe de façon suffisamment large pour que les étudiants aient la possibilité de les infléchir en fonction de leurs besoins propres ou de leurs préférences. Enfin, on peut espérer que cela les conduira à développer leur métier d'apprenant par un travail en classe et hors de la classe.

\section{Expérimentation de la démarche en licence AES}

21 Pour donner un aperçu de la démarche que nous proposons, nous nous référons ici aux stratégies d'enseignement adoptées cette année en licence AES. Les cours avaient lieu pendant un seul semestre (le premier), à hauteur d'une heure et demie par semaine, soit un total de $15 \mathrm{~h}$ de cours et $3 \mathrm{~h}$ d'évaluation. Le groupe était constitué de 35 étudiants (35 étudiants inscrits, 33 en fin de parcours). Les cursus d'amont étaient très diversifiés.

\subsection{Analyse préalable et choix didactiques}

Nous reprenons ici la première étape du cheminement didactique : des observations dans la situation d'enseignement-apprentissage à la définition d'objectifs qui en découle.

\subsection{1. État des lieux et remédiations envisageables}

23 Dès le tout début de la première séance, outre la taille du groupe, il est remarquable que le problème majeur soit celui de l'hétérogénéité des niveaux: la relative aisance de quelques étudiants contraste avec les difficultés de la grande majorité et se traduit d'ailleurs par un refus de prise de parole pour quelques-uns. Nous en déduisons que le dispositif devra permettre une individualisation des travaux des étudiants; cela impliquera que le cours y contribue en servant de point d'appui pour des activités dans la langue qu'ils auront à gérer en grande partie en autonomie pour accroître sensiblement la pratique de la langue.

24 Nous remarquons également que l'attitude passive qui prévaut dénote un manque de confiance quasi général qui se traduit par une attente de «bonnes réponses ». Ainsi, dans le travail donné pour cette première séance, le blocage intervient à deux niveaux : à l'écrit pour la compréhension des données factuelles et chiffrées d'un article et à l'oral pour la restitution de quelques données. Il en ressort que la mise en confiance doit être un élément clé du dispositif qui devra leur donner des moyens d'agir avec la langue dans des situations perçues comme ne mettant pas personnellement en danger. Rappelons ici que l'apprentissage d'une langue étrangère suppose le passage d'un système de représentations à un autre: en impliquant ainsi la personne tout entière, cet apprentissage est souvent très déstabilisant (Mallet 2003). L'attitude et la motivation sont donc au cœur des préoccupations didactiques et nous pensons qu'elles doivent orienter la création de dispositifs.

\subsubsection{Définition d'objectifs}

Pour le cadrage didactique du paysage professionnel dont nous avons tracé les grandes lignes, la définition d'objectifs suppose un choix dans la prise de vue qui mettra en avant des traits caractéristiques retenus pour leur pertinence en regard les améliorations visées: ici, un développement substantiel de la pratique et une mise en confiance progressive. Nous optons pour la recherche documentaire et son exploitation sous forme 
d'exposé succinct, mettant ainsi l'accent sur le développement de la compréhension écrite et de l'expression orale. La situation qui nous sert de point d'appui est la participation à un événement professionnel (cf. Annexe 1) au cours duquel la personne devra intervenir oralement; il pourra s'agir d'une réunion de travail, d'un colloque ou d'un séminaire. Dans ce contexte, la situation de communication suppose en amont un travail sur des supports écrits pour une bonne connaissance du dossier: il faut notamment parcourir une documentation abondante de façon sélective pour en extraire des éléments pertinents, relever des éléments et/ou en dégager l'essentiel.

D'autre part, nous émettons l'hypothèse que, pour développer conjointement la motivation et la pratique, les consignes pour leur recherche doivent encourager l'utilisation d'Internet sans exclure les sources traditionnelles. Nous invoquons deux raisons principales : l'aspect quantitatif et actuel des données qu'ils auront à traiter en fonction de choix personnels, et d'autre part, suivant en cela une définition située de l'apprentissage (Lave \& Wenger 1991), l'impact prévisible sur les processus cognitifs à l'œuvre de la situation spécifique d'enseignement-apprentissage générée par la quête de données sur Internet.

\subsection{Aspects de la mise en forme didactique}

L'organisation par tâches comporte deux parties: un travail collaboratif qui vise le développement progressif des savoirs et savoir-faire dans des tâches intermédiaires ainsi qu'une tâche plus globale permettant de vérifier le cheminement individuel.

\subsubsection{Des tâches d'entraînement}

Les activités prennent appui non pas sur des textes mais sur des dossiers composés d'éléments de l'actualité sociale et économique prélevés sur Internet et suffisamment denses pour présenter plusieurs points de vue sur des phénomènes récents. Nous avons privilégié une organisation thématique pour une familiarisation avec le lexique et les problématiques afférents.

Le guidage vise avant tout la fluidité et requiert un entrainement donnant les moyens de se faire comprendre. Dans la première séance consacrée à la recherche d'informations contradictoires, chaque groupe dispose de documents différents mais complémentaires. La mise en commun par le premier rapporteur met d'emblée l'accent sur des déficits précis dans la communication orale. Ce sont ces points qui sont travaillés en priorité dans les séances suivantes: préparation des grandes lignes de l'argumentation à la maison, mise en commun à l'intérieur de chaque groupe avec grille d'analyse de la qualité de la communication, la prise de parole en autonomie n'intervenant qu'ensuite.

D'autre part, l'entrainement concerne la recherche documentaire en focalisant sur les mises en perspective et sur le recul critique pour déterminer ce qui sera pertinent ou non dans la présentation orale. En conséquence, les supports sont soumis aux étudiants en l'état, quels que soient leur forme, leur longueur et leur niveau de difficulté. La didactisation intervient alors principalement pour étayer les prises de risques dans les choix et pour organiser les apports méthodologiques. Ainsi, sur le dossier intitulé «Who's to blame for trade talk collapse?» qui comportait la totalité d'un forum de discussion ${ }^{1}$, chaque groupe d'étudiants a eu à sélectionner des arguments dans une partie du document qui avait préalablement été découpé arbitrairement ; cette recherche a servi de 
base à une discussion avec les autres groupes pour un classement en fonction de critères qu'ils devaient expliciter. D'autres besoins méthodologiques ont ensuite pu être traités, notamment l'organisation des données dans une synthèse et leur mise en forme sur un transparent servant de point d'appui à la présentation. Les moyens à développer pour une bonne qualité de la communication orale devant un public devenaient ainsi presque naturellement nécessaires.

\subsubsection{Une tâche en autonomie}

31 L'évaluation certificative s'est fondée sur une tâche plus globale. Il s'agissait de l'élaboration d'un dossier par groupe (deux à trois étudiants) servant de point d'appui à une présentation orale individuelle. Le thème était libre mais il devait avoir trait aux domaines économiques et sociaux ; les éléments du dossier devaient être récents (moins d'un an) et provenir de sources anglophones.

Durant l'exposé, chacun des membres du groupe présente un volet spécifique du thème et/ou de la problématique choisi(e). Les notes ne sont pas autorisées mais il est possible de prendre appui sur un transparent. Deux questions sont ensuite posées pour chaque intervenant. La notation de la présentation orale s'appuie sur la grille d'évaluation élaborée en cours pendant la phase d'entraînement ; elle comporte trois volets : qualité de la communication (audible, compréhensible, intéressante, soucieuse du public,...), traitement du sujet choisi (contenus, sélection d'items,...) et aspects méthodologiques (organisation, argumentation,...). Il s'agit donc essentiellement d'être compris et aussi d'être crédible et, par conséquent, la qualité de l'anglais n'intervient que dans ce cadre. De la même façon, l'évaluation du dossier tient compte de son intérêt pour la personne qui le consulte (pertinence des sélections opérées, organisation et mises en perspective des données,...). Le bilan d'ensemble a été très encourageant: les progrès ont concerné aussi bien la qualité de la communication en anglais que l'attitude et la motivation.

\subsubsection{Bref retour sur l'expérimentation}

Pour bénéficier du point de vue des étudiants, un premier questionnaire leur a été distribué (cf. Annexe 2). Il a été renseigné anonymement lors de la remise des dossiers (quelques semaines avant les exposés oraux). Il visait avant tout l'exploration de la démarche de recherche documentaire, mais nous retiendrons ici plus particulièrement la façon dont ils ont vécu ce type de travail en nous penchant sur la question "Quel intérêt avez-vous retiré de cette recherche?» (cf. Exemplier en Annexe 3). Prenons le cas d'un premier étudiant $\mathrm{E} 1$ : «J'ai lu des textes en anglais, ce que je fais rarement. J'ai appris à mieux connaître certains membres du groupe ». Ceci nous semble emblématique des remarques des étudiants, pour lesquels l'intérêt de la recherche concerne principalement deux aspects. D'une part, ils évoquent des progrès dans le travail dans la langue sous des aspects quantitatifs (E1) et parfois qualitatifs comme le souligne par exemple E2: « intérêt pour le thème. La lecture de revues étrangères permet une 'prise de recul' par rapport à l'environnement national ». D'autre part, le travail de groupe les a aidés ; citons à ce sujet E3 : «Intérêt pratique et méthodologique car ça nous habitue à travailler en groupe sur des sujets variés ». Ces quelques éléments semblent corroborer l'idée qu'il convient d'accorder une importance toute particulière aux mises en situation dans l'élaboration de tâches et aux apports potentiels d'un travail collaboratif. 
second dispositif de recueil de données sur l'expérimentation a été mis en place à l'issue des exposés oraux ; les étudiants devaient s'exprimer librement et anonymement sur leur prestation et faire des propositions pour améliorer le cours de l'année prochaine. À titre d'exemple, voici ce qu'écrit $\mathrm{E} 4$ : « la mise en forme de mon passage à l'oral : j'ai dû apprendre à m'exprimer avec un vocabulaire adapté à l'oral et non utiliser un vocabulaire d'écrit». La prise de conscience de la spécificité de la communication orale semble bénéficier de mises en situation dans une approche par tâches. Pour terminer citons les améliorations proposées par $\mathrm{E} 5$ : «c'est une approche intéressante de la langue anglaise plus je pense que des tests de grammaire écrits. Il faudrait davantage étudier en cours les 'petits trucs' de communication. Je pense que l'expérience doit être renouvelée voire considérée comme examen typique de notre niveau d'étude ».

\section{Conclusion}

La discussion sur l'intégration de la professionnalité dans l'enseignement-apprentissage de la langue de spécialité suppose la prise en compte de la complexité qui intervient à plusieurs niveaux dans la langue des différents milieux professionnels. L'approche didactique ajoute systématiquement à cette complexité en insérant des positionnements et donc des regards qui sont portés sur la langue dans des situations professionnelles. La difficulté que l'on rencontre alors est stratégique et paradoxale : il nous faut, à partir de descriptions de cette réalité composite et problématique trouver les moyens de faire progresser les étudiants. En résumé, la question majeure est celle de la simplification didactique pour laquelle nous devons garder à l'esprit la spécificité de notre public de grands apprenants. Nous avons pu observer que l'élaboration de tâches se fondant sur une analyse des situations de communication pouvait contribuer favorablement à une prise en compte d'un espace professionnalisé. Il nous semble alors particulièrement important d'associer les étudiants à ce travail en leur donnant des tâches dans des situations suffisamment ouvertes pour qu'ils en comprennent mieux les enjeux par rapport à leur propre expérience d'apprenant.

\section{BIBLIOGRAPHIE}

Aranda, E. 2002. « Dynamiser les apprentissages dans un contexte institutionnel ». ASp 37-38, 89-94.

Bailly, A. \& R. Ferras. 1996. Éléments d'épistémologie de la géographie.Paris :Armand Colin.

Brown, J., A. Collins \& P. Duguid, 1989. « Situated cognition and the culture of learning ». Educational Researcher 18, 32-42.

Daley, E. 2003. « Expanding the concept of literacy, the internet and the university: Forum 2002 » In Devlin, M., R. Larson \& J. Meyerson (dir.), A joint project of the Forum for the Future of Higher Education and Educause <http://www.educause.edu/ir/library /pdf/ffpiu027.pdf>. 
Delevotte, C. 2003. «Accès aux ressources documentaires en situation d'autonomie : pratiques et représentations ». Alsic 6/1,45-63 <http://www.alsic.org/>.

Deyrich, M-C. 2001. «Quelles médiations pour une gestion efficace de la transposition didactique en anglais de spécialité ?». ASp 31-33, 143-152.

Dressen Hammouda, D. 2003. « Contributions of an integrated genre theory of text and context to teaching LSP ». ASp 39-40, 73-90.

Ellis, R. 2003. Task-based Learning and Teaching. Oxford : Oxford University Press.

Karsenti, T. \& F. Larose (dir.). 2001. Les TIC... au cour des pédagogies universitaires : diversité des enjeux pédagogiques et administratifs. Sainte-Foy (Québec, Canada) : Presses de l'université du Québec.

Lave, J. \& E. Wenger. 1991. Situated Learning: Legitimate Peripheral Participation. Cambridge : Cambridge University Press.

Linard, M. 2000. «Les TIC à l'université : potentiel des outils et conditions d'accès à l'autonomie de la connaissance », communication aux Journées@univ, 11-13 octobre 2000, Orme-Marseille $<$ http://www.educnet. education.fr/chrgt/linard.rtf>.

Lyotard, J. F. 1979. La Condition postmoderne : Rapport sur le savoir. Paris : Éditions de Minuit.

Mallet, B. 2003. « La Langue mise en bouche ». ELA 131, 291-303.

Maulini, O. 2000. L'École simulée ? La forme scolaire face aux environnements virtuels : conséquences pour la transposition dans le contrat didacTIC. Faculté de Psychologie et des Sciences de l'éducation, Université de Genève <www.unige.ch/fapse/SSE/teachers/maulini/simul.html>.

Mémet, M. 2003. «L'Enseignement à contenu intégré augmente la motivation pour l'apprentissage de la langue : vrai ou faux ? Étude portant sur des cours d'anglais de spécialité en médiation culturelle ». ASp 39-40, 131-142.

Resche, C. 2003. « Décryptage d'un genre particulier : les communiqués de presse de la Banque centrale américaine ». ASp 39-40, 21-35.

Resnick, M. 2002. « Rethinking learning in the digital age ». In Dutta, S., Lanvin, B.\&F. Paua (dir.), The Global Information Technology Report 2001-2002. Readiness for the Networked World. Oxford : Oxford University Press2002 World Economic Forums Reports.

Ricœur, P. 2004. Parcours de la reconnaissance. Paris : Stock.

Swales, J. M. 1990. Genre Analysis. Cambridge : Cambridge University Press.

Tardif, J. 1998. Intégrer les nouvelles technologies de l'information. Quel cadre pédagogique? Paris : ESF.

Vygostski, L. S. 1985. Pensée et langage.coll. Terrains. Paris : Messidor.

Widdowson, H. G. 2003. Defining Issues in English Language Teaching. Oxford : Oxford University Press.

\section{ANNEXES}

\section{Annexe 1}

Interprétation didactique du paysage professionnel en AES 


\begin{tabular}{|c|c|c|}
\hline & Pratiques langagières & Savoir-faire \\
\hline $\begin{array}{l}\text { information } \\
\text { liée à l'actualité } \\
\text { économique }\end{array}$ & $\begin{array}{l}\text { lire la presse afin de: } \\
\text { - compléter l'état des connaissances donc de } \\
\text { relever les éléments pertinents } \\
\text { - prendre position }\end{array}$ & $\begin{array}{l}\text { Compréhension } \\
\text { écrite } \\
\text { (expression écrite) } \\
\text { (expression orale) }\end{array}$ \\
\hline $\begin{array}{l}\text { DOCUMENTATION SUR LE } \\
\text { MONDE ÉCONOMIQUE }\end{array}$ & $\begin{array}{l}\text { parcourir une documentation abondante de } \\
\text { façon sélective pour en extraire des éléments } \\
\text { pertinents }\end{array}$ & $\begin{array}{l}\text { Compréhension } \\
\text { écrite } \\
\text { (expression orale) } \\
\text { (expression écrite) }\end{array}$ \\
\hline $\begin{array}{l}\text { recherche } \\
\text { documentaire }\end{array}$ & $\begin{array}{l}\text { relever des éléments et/ou } \\
\text { en dégager l'essentiel }\end{array}$ & $\begin{array}{l}\text { Compréhension } \\
\text { écrite } \\
\text { (expression écrite) } \\
\text { (expression orale) }\end{array}$ \\
\hline $\begin{array}{l}\text { Participation } \\
\text { À DES ÉVÉNEMENTS } \\
\text { professionnels }\end{array}$ & $\begin{array}{l}\text { intervenir (à des degrés divers) dans des } \\
\text { réunions de travail, des colloques ou des } \\
\text { séminaires, implique souvent: } \\
\text { - un travail de préparation, } \\
\text { - l'élaboration de supports visuels }\end{array}$ & $\begin{array}{l}\text { Expression orale } \\
\text { (compréhension } \\
\text { écrite) } \\
\text { (compréhension } \\
\text { orale) } \\
\text { (expression écrite) }\end{array}$ \\
\hline
\end{tabular}

\section{Annexe 2}

\section{Questionnaire}

\section{RECHERCHE MENÉE POUR L'ÉLABORATION D'UN DOSSIER SERVANT DE SUPPORT A UN} EXPOSÉ ORAL

\section{a) la recherche}

Par où avez-vous débuté ? Quelles étapes avez-vous suivies?

Quels sites (livres, journaux) avez-vous regardés? Qu'avez-vous conservé comme information?

Comment avez-vous décidé du thème? du plan?

Quels problèmes avez-vous rencontrés?

Comment s'est déroulé le travail de groupe?

Quel intérêt avez-vous retiré de cette recherche?

\section{b) renseignements personnels}

F/M âge

situation familiale

Lieu de résidence (parents, appartement d'étudiant...)

Langues parlées

Études déjà menées

c) sources d'information

Journaux lus (fréquence) 
Informatique : quel équipement? (personnel ?) quel usage?

utilisation d'Internet? fréquence ? Domicile ou ailleurs ?

d) remarques complémentaires

\section{Annexe 3 \\ Exemplier}

\section{1 - Réponses au questionnaire (cf. Annexe 2)}

Question : Quel intérêt avez-vous retiré de cette recherche?

E1 : «J'ai lu des textes en anglais, ce que je fais rarement. J’ai appris à mieux connaittre certains membres du groupe ».

E2 : « Intérêt pour le thème. La lecture de revues étrangères permet une 'prise de recul' par rapport à l'environnement national ».

E3 : « Intérêt pratique et méthodologique car ça nous habitue à travailler en groupe sur des sujets variés».

\section{2 - Expression libre sur leur prestation et propositions pour améliorer le cours de l'année prochaine}

E4 : « La mise en forme de mon passage à l'oral : j'ai dû apprendre à m'exprimer avec un vocabulaire adapté à l'oral et non utiliser un vocabulaire d'écrit ».

E5: «C'est une approche intéressante de la langue anglaise plus je pense que des tests de grammaire écrits. Il faudrait davantage étudier en cours les 'petits trucs' de communication. Je pense que l'expérience doit être renouvelée voire considérée comme examen typique de notre niveau d'étude ».

\section{NOTES}

1. Sur le site <www.bbc.com>, dans la rubrique " BBC News, Have your say".

\section{RÉSUMÉS}

L'article s'intéresse à la notion de «milieu professionnel » pour tenter d'envisager dans quelle mesure elle peut contribuer efficacement à la définition de contenus d'enseignementapprentissage pour l'anglais de spécialité à l'université. Pour tenir compte du contexte et dans la perspective théorique de la cognition située, l'auteur propose de passer de la notion de milieu à celle de "paysage professionnel ». On examinera comment, à partir des savoir-faire langagiers jugés utiles dans des circonstances professionnelles spécifiques, ce cadrage devrait permettre d'élaborer des tâches d'enseignement-apprentissage conçues pour le développement d'une méthodologie transférable. Cette approche sera ensuite illustrée dans une interprétation d'un espace didactique professionnalisé en licence AES. Pendant un semestre, les tâches ont impliqué un processus de didactisation et des techniques de guidage combinant, d'une part, l'entraînement méthodologique à la pratique de la communication orale et, d'autre part, l'entraînement à la recherche de données et à leur exploitation en autonomie. 
The article investigates the notion of "professional domain" in order to assess how it can contribute efficiently in a personalized definition of learning and teaching objectives and contents of ESP at university level. Bearing the context in mind, and within the theoretical framework of situated cognition, a shift from the notion of domain to that of "professional landscape" is suggested. This frame of reference is then examined to check its potential to highlight the language skills deemed relevant in specific professional circumstances. The ensuing objective is to explore the possibility of task design aiming at the development of transferable methodology. The approach is then exemplified by a didactic interpretation of professional contextualisation in Licence AES. During one semester, task planning included both didactic transformations and scaffolding techniques, thus enhancing methodological practice in oral communication skills as well as training in autonomous information research retrieval and exploitation.

INDEX

Keywords : language for specific purposes, learners' training, task design, teaching strategy Mots-clés : élaboration de tâches, entraînement dans l'apprentissage, langue de spécialité, stratégie d'enseignement

\section{AUTEUR}

MARIE-CHRISTINE DEYRICH 